\title{
Mediterraneanisms and Colloquial Maritime Terminology in Croatia
}

\author{
Dr.Sc. Jasenka Maslek \\ University of Dubrovnik, Croatia \\ E-mail: jasenka.maslek@unidu.hr \\ Ariana Violić-Koprivec \\ University of Dubrovnik, Croatia \\ E-mail: ariana.violic-koprivec@unidu.hr
}

\section{Doi:10.5901/mjss.2013.v4n10p119}

\begin{abstract}
The course and direction of development of maritime terminology on the eastern Adriatic coast followed the rhythm of socioeconomic changes and stronger or weaker trade relations with the Mediterranean countries, especially with the Italian maritime republics. Thus, the impact of foreign languages is greater in the maritime terminology than in any other field. Croatia, i.e. its coast, has through centuries been a part of various social and political systems. It was not before the mid 19th century that the national awareness and integrative tendencies became stronger, hence the need for education in the mother tongue. Thus began the systematization of the Croatian maritime terminology. Its development will be accompanied by the coexistence of the two terminology systems, standardized and colloquial terms. Colloquial terms are still widely used in the language of our seafarers and seaside population due to the centuries-long trade relations with the Mediterranean countries. This paper describes the circumstances leading up to the development of the maritime terminology in Croatia, France and Italy, countries that had dynamic trade relations and direct maritime contacts. The similarity among the Croatian maritime idioms, Italian dialects, and French terminology mostly dates back to the time of sailing, whereby the commands and terms for parts of ship, ropes and rigging stand out. For the selected colloquial terms that are still, more or less frequently, used in the eastern Adriatic coast, and that originate from the given time, it will be determined whether there are their equivalents in French and Italian in the 18th and 19th century sources (the period of an intensive contact among these countries).
\end{abstract}

Keywords: mediterraneanisms, croatian maritime terminology, colloquial terminology, maritime dictionnaries

\section{Introduzione}

"La comunicazione continua reciproca delle culture diverse è la caratteristica principale dell'identità del Mediterraneo" (Bertoša, 2002: 47). La mentalità mediterranea croata è il risultato del modo di vivere e lavorare sulla costa adriatica $\mathrm{e}$ nel suo entroterra, dei popoli che sono stati per secoli in contatto reciproco continuo, ovvero della compenetrazione di mondi e culture diverse. „In questo modo, la particolare mentalità mediterranea si è adattata al mondo dell'uomo croato, abitante della costa adriatica" (Bertoša, 2002: 44). I marinai croati navigarono per secoli, non solo nel Mediterraneo orientale e nel Levante, ma visitarono anche le sue coste occidentali, i porti della Francia, della Spagna, del Portogallo e anche dell'Inghilterra e dell'Olanda. Con l'andare dei secoli si sono sviluppati collegamenti reciproci, costruite relazioni e conosciute tradizioni, culture e lingue specifiche (Bertoša, 2002: 19-25).

Lo sviluppo di termini marinari sulle sponde orientali dell'Adriatico pulsava al ritmo di cambiamenti socio-economici e del rafforzamento o cessamento degli scambi commerciali con i paesi del Mediterraneo, particolarmente con le repubbliche marinare italiane e con altre regioni della penisola appenninica. Nel lessico marittimo è quindi, più che nelle altre attività, presente l'influsso delle lingue straniere. „I contatti linguistici sono sempre il risultato di un contatto storico e culturale, così gli influssi croato-romani e le loro compenetrazioni, fin dall'inizio della storia croata sulla costa adriatica orientale, aprirono la strada verso la cultura mediterranea, una delle componenti più importanti dell' identità culturale croata (Sočanac, 2002: 127).

I contatti delle lingue provocarono prestiti linguistici. Le conseguenze di questi contatti possono variare dal prestito lessicale superficiale, attraverso varie forme di influenza sulla struttura del linguaggio, fino agli influssi strutturali profondi (Sočanac, 2002: 127, secondo Thomason \& Kaufman, 1991). Blommfield (1970) distingue i prestiti culturali e quelli intimi. II prestito intimo è determinato dal contatto diretto e dalla comunicazione orale, mentre la formazione scolastica 
dell'interlocutore non è decisiva. Succede, a causa dell'uso simultaneo di più lingue nella stessa area geografica, che la cosiddetta lingua „superiore” diventi fonte di prestito per la lingua la cui posizione sociolinguistica è inferiore. In questo caso si ha il vero e proprio prestito intimo.

Nella marineria croata dell'Ottocento la lingua ufficiale era l'italiano e le lingue d' insegnamento nelle scuole, oltre all'italiano, erano il tedesco e l'ungherese. ${ }^{1}$ Nella seconda metà dell'Ottocento ci fu una tendenza di rafforzamento della coscienza nazionale e della necessità di standardizzazione della terminologia specialistica croata. Si verificò anche il rifiuto di prestiti linguistici prevalenti e dell'influenza linguistica straniera nella terminologia marinara. In questo contesto va notata la necessità dell'insegnamento nella lingua materna. Inizia così a sistematizzarsi la terminologia marinara croata. II suo sviluppo sarà accompagnato dalla coesistenza di due sistemi terminologici - terminologia standardizzata e usuale (Stolac, 1998). Termini usuali sono presenti, con maggiore o minore frequenza, anch'oggi nella parlata dei nostri marinai e abitanti delle coste, come conseguenza di scambi commerciali plurisecolari con i paesi del Mediterraneo.

Questo articolo offre una panoramica delle circostanze in cui prosperava il croato, ma anche la terminologia marinara specialistica dei paesi con i quali la costa adriatica orientale per secoli era stata legata, come la Francia e I'Italia. Inoltre, per i termini colloquiali selezionati appartenenti al tempo della navigazione a vela e legati ai comandi e ai nomi delle parti della nave, corda e alberatura, verrà stabilito se ci sono $i$ loro equivalenti in francese e in italiano nelle fonti del Settecento e dell'Ottocento, quindi al momento dei loro contatti molto intensi.

Lo scopo del presente lavoro è quello di mostrare che la terminologia marinara colloquiale in Croazia appartiene ai nostromismi ${ }^{2}$ i quali sono in gran parte anche mediterraneismi che si utilizzarono per secoli nel Mediterraneo. Anche in Croazia, questa terminologia esiste come risultato del suo uso plurisecolare ed è viva ancora oggi nella parlata marinara dalmata. Tuttavia, la terminologia colloquiale appare raramente nella lessicografia marittima croata (Božanić et al. 2011). Pertanto, sembra logica la conclusione di Diana Stolac (1998: 155) che "la coesistenza secolare di due sistemi terminologici richiedeva una specifica riconciliazione" e che si è giunti al punto di registrare, oltre ai termini standardizzati, anche quelli usuali.

\section{Opzioni decisive di sviluppo della terminologia marinara croata}

La crescenza di una coscienza nazionale nel corso dell'Ottocento portò, tra le altre cose, alla necessità di creare una nuova terminologia marinara croata. A questo fine con tutto il cuore si dedicò Božo Babić, professore dell'Istituto nautico a Bakar e autore del primo dizionario marittimo stampato in Croazia. ${ }^{3}$ Dal 1870 al 1901 egli pubblicò cinque dizionari marinari lasciando così un segno estremamente significativo nella lessicografia marittima croata. Questi sono cinque libretti sulla tematica marittima, che sebbene a prima vista non indichino il lavoro lessicografico, sono tuttavia considerati dizionari ${ }^{4}$ (D. Stolac, 1998: 49., Stepanić, 2006: 70). Durante lo sviluppo della terminologia marittima croata si

\footnotetext{
1 La lingua croata nell'istruzione nautica, anche se brevemente, per la prima volta cominciò ad essere utilizzata a Fiume verso la metà dell'Ottocento. In questo sforzo particolarmente si sono affermati gli insegnanti dell'Istituto nautico a Fiume i quali, insieme alla parte dell'intelligenza croata di Fiume, cercarono di rimuovere dalla lingua croata non solo i frutti della dominazione straniera, ma anche ciò che per secoli, per via naturale, entrò nella lingua croata dal circolo marittimo del Mediterraneo (Stulli, 1953: 74). Intorno al 1870 tendenze simili sono evidenti, come già menzionato, nell'Istituto nautico a Bakar. II suo direttore e professore Božo Babić per più di trent'anni cercò di raccogliere e creare la terminologia marinara croata promuovendo nello stesso tempo le tendenze puristiche radicali (Stepanić, 2006: 71).

2 "Così, nella parlata dei nostri vecchi marinai prevalsero gli italianismi. Alcuni linguisti denominano nostromismi quelli che sono rimasti vivi ancora oggi e fanno parte attiva del loro lessico colloquiale" (Stepanić, Violić-Koprivec i Maslek, 2009: 226 secondo Urbany, 1994 e Stolac, 1998). II nome deriva dall' italianismo nostromo. Nostromismi sono prestiti che sono stati completamente assimilati e adattati alla lingua croata a livello fonologico e morfologico con vari gradi di adattamento alle parlate particolari o dialetti (Urbany, 1996: 205-208). Romanisti stranieri li classificano come est Venetian words (Stepanić 2005: 168 secondo Kahane 1953). A causa della somiglianza del sistema fonologico delle due lingue è significativamente ridotta la necessità di adattamento fonologico ed è cresciuta la possibilità di prendere il prestito senza difficoltà a pronunciarlo. In tal modo la parola straniera era stata pronunciata con gli elementi fonologici esistenti nella parlata del destinatario.

3 Anche prima di questo vocabolario in alcune opere letterarie e dizionari croati apparvero termini legati alla terminologia marinara, come ad esempio nelle opere di Faust Vrančić nel 1595 e Pavle Ritter Vitezović verso la fine del Seicento (Stolac, 1998).

${ }^{4}$ Due di loro, il primo e l'ultimo, sono dizionari marinari universali che incorniciano un periodo di intenso impegno di Babić nella terminologia marinara croata e i suoi sforzi persistenti per introdurre la lingua croata tra le lingue marinare dell'Impero Austro-Ungarico. Il suo primo dizionario è Morski riečnik hrvacko-srbski usporedjen s italijanskijem jezikom od jednoga pomorca, iz 1870. godine (Dizionario di marina croato-serbo paragonato con la lingua italiana da un marinaio, del 1870). L'ultimo dizionario di Babić, Pomorski rječnik ili nazivlje za brodarenje po moru (Dizionario di marina o terminologia della navigazione) stampato nel 1901, è il risultato di una
} 
intrecciarono due correnti prevalenti, quella del purista Božo Babić che, dopo aver respinto tutti gli italianismi e forestierismi, ha introdotto una formazione completamente nuova, e quella tradizionale, scettica alle novità, purismo linguistico e neologismi (Vidović, 1982: 796), a cui apparteneva Juraj Carić, collega di Babić dell'Istituto nautico a Bakar.

Carić tendeva al mantenimento degli italianismi radicati credendo che molte espressioni marittime, anche se prima sembravano parole straniere, successivamente avevano preso la forma croata e dopo l'adattamento completo avevano perso le caratteristiche dei prestiti (Stepanić, 2006: 72). L'autore si riferisce ai già nominati nostromismi. Ecco perché non c'è bisogno di ripulire il linguaggio marinaro dagli italianismi, considera Carić, la cui opinione è sostenuta da Pero Budmani che dichiara che è „barbarismo minore ricevere una parola straniera piuttosto adattare la nostra ad un'altra lingua." Perciò Carić non ritiene opportuno insegnare nelle scuole secondo la terminologia creata, che non viene utilizzata e quindi non si capisce nemmeno nei libri (Carić, 1890: V).

Cosí anche il famoso linguista Hugo Schuchardt dice che non esiste una lingua che non sia "mescolata”. Nella sua opera Slawo.Deutsches und Slawo.Italienisches si occupa della situazione linguistica all'interno dell'Impero asburgico, specialmente nelle aree di contatto del mondo slavo, germanico e romano. II suo atteggiamento verso i contatti linguistici è positivo ed è contrario al purismo, che allora era caratteristico per alcuni ambienti slavi. Dall'altra parte, Schuchardt critica il „falso senso di orgoglio nazionale per cui dalle lingue slave sono stati eliminati molti prestiti e con loro anche le traccie della storia di queste popolazioni e le loro relazioni con i vicini" (Sočanac, 2002: 129 secondo Gusmani, 1995: 251-263).

Vanno ricordati anche gli sforzi per creare un vocabolario specialistico per mezzo dei decreti dopo la seconda guerra mondiale, ma che non hanno portato ai risultati desiderati. Un esempio è la proposta della Società per la Ricerca e il miglioramento della marineria jugoslava del $1973 .{ }^{5}$ E stato un richiamo alla coerente applicazione di termini tecnici nazionali, con lo scopo di purificare la lingua dalle parole prese in prestito, imponendoli e obbligando al loro uso. II reparto della marineria fa appello in questo senso alle scuole, stampa e TV, mentre per altri partecipanti, ritengono, dovrebbe essere obbligatoria la pratica dei termini adottati (cioè, selezionati, coniati). „Per questo i termini adottati devono essere propagati in tutte le riviste marittime mentre gli organi politico-sociali della nave vanno impegnati al controllo dell'applicazione dei termini nazionali nel lavoro quotidiano sulla nave" (Pomorstvo, god. XXVIII broj 5-6, 1973: 186).

\section{Romanismi nell'espressione marinara della Dalmazia}

La Dalmazia è per secoli stata governata dalla Repubblica di Venezia ed è comprensibile il suo impatto significativo sul campo lessicale e linguistico nelle parlate marinare locali. Nel corso dei secoli, sulla sponda orientale dell'Adriatico si parlava dalmatico, lingua romanza in Dalmazia (Viarello, 1955), come uno stadio intermedio tra il latino e il romanzo. II dalmatico era in contatto costante con le parlate italiane volgari e perciò il periodo di maggiore influenza del veneziano è registrato sullo strato già romanizzato. Questo processo ha avuto luogo durante la seconda metà del Quattrocento (Viarello, 1955). Del contatto diretto delle parlate locali costiere e della lingua veneta, sulla costa dalmata risulta la formazione della lingua coloniale veneziana. Così la maggior parte della terminologia marinara croata dialettale deriva direttamente dalla lingua veneta, mentre la parte minore (di origine greca, latina, spagnola, araba) è venuta indirettamente, anche attraverso il veneziano. ${ }^{6}$ „Dialetti veneziani sulla sponda adriatica orientale possono essere chiamati coloniali perché non sono lingue romanze autoctone, ma sviluppate sul substrato romano e adstrato slavo"(Stepanić 2011: 165 secondo Sočanac, 2004: 89).

Siccome non si tratta di un'interferenza tra due lingue simili, ma di lingue appartenenti a gruppi geneticamente diversi, romanzo e slavo, il loro intreccio ha generato numerosi prestiti linguistici. Essendo accentuata la diversità tipologica del croato come lingua sintetica con un ordine libero delle parole, e l'italiano, una lingua parzialmente analitica

lunga ricerca delle migliori soluzioni nella terminologia marinara croata ed è il punto culminante del lavoro lessicografico di Babić (Stepanić, 2006: 71).

5 La riunione consultiva sulla terminologia marinara si è tenuta a Spalato il 22 marzo 1973 per iniziativa della Sezione marinara della Società per la promozione della marineria jugoslava. Questo convegno è stato ispirato dal fatto che sulle navi nazionali costantemente, e sembra più che prima, venisse protetta la terminologia straniera. A questa pratica è soggetto, sotto l'influenza degli ufficali e marinai anziani, anche personale giovane e qualificato che è costretto a dimenticare le espressioni imparate a scuola e ad accettare la distorta terminologia straniera (Pomorstvo, god. XXVIII, broj 5-6, 1973: 185). Dal testo è abbastanza chiaro che sotto le espressioni interne imparate a scuola sia implicata l'appena creata terminologia utilizzata nelle scuole.

6 Žarko Muljačić si è interessato alla ricerca dei romanismi di origine araba che sono arrivati nella nostra lingua attraverso il veneziano coloniale 0 arabismi italiani del sud Italia. A causa della loro natura indiretta l'autore li denomina pseudoarabismi (Muljačić, 2007). 
con un ordine definito di parole nella frase, i loro contatti diventano tanto più interessanti ed evidenti, col risultato di veri e propri prestiti linguistici (Lovrić-Jović, 2006). I risultanti prestiti veneziani e italiani nella terminologia marinara croata sono la conseguenza dei contatti linguistici diretti, cioè il risultato della comunicazione naturale (Jernej, 1956). Ciò è confermato anche nel saggio di Mirko Deanović che cita il Mediterraneo come uno spazio di interazione diretta delle lingue, delle culture e delle civiltà, e la cui particolarità si riflette nella diversità di esistenza e sopravvivenza alla base dell'elemento comune - il mare (Deanović, 1962). Quindi non è sorprendente che proprio la terminologia marinara nella zona adriatica orientale sia basata sull'influenza del dialetto veneziano, ma anche su molti mediterraneismi universali. ${ }^{7}$ Non ci si sarebbe potuto aspettare altrimenti lungo la costa croata che apparteneva a diversi sistemi sociali e politici, sempre al servizio degli altri, e fino al tempo della rinascita verso la metà dell'Ottocento senza tendenze nazionali integrative. La mancanza dello stato perciò si riflette sull'inesistenza di un linguaggio e di una terminologia marinara nazionale.

\section{II lessico marittimo in Francia e in Italia}

I paesi del Mediterraneo che sono stati indissolubilmente legati per secoli, in particolare l'Italia e la Francia, hanno un diverso contesto storico entro il quale si è sviluppata la loro terminologia marinara. Nel corso dei secoli, la terminologia francese ha subito diverse influenze linguistiche da latino, greco, lingue scandinave, olandese, arabo, italiano, fino alla lingua inglese nell'Ottocento. Già dal dodicesimo secolo in testi scritti in francese antico è stato registrato l'uso di termini nautici che fa riferimento ad un vocabolario specifico relativo alla gente del mare. Marc Van Campenhoudt inizia a presentare lo sviluppo della terminologia marinara francese citando Villain-Gandossia (1999: 24): „La terminologia della navigazione, per definizione più internazionale che qualsiasi altro vocabolario tecnico, è cresciuta in base a numerosi

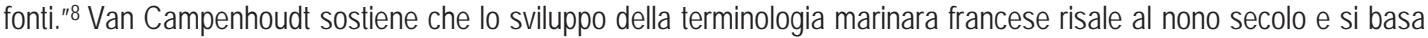
sul patrimonio greco, latino, germanico e arabo. Le influenze particolarmente importanti sul suo sviluppo risalgono al periodo delle Crociate e delle grandi scoperte geografiche. E mentre l'evoluzione della lingua francese si stava muovendo verso la sua unificazione, la terminologia marinara progrediva in due direzioni diverse, che hanno portato all'esistenza di due lingue quasi completamente diverse: una in uso nelle galere e l'altra sulle barche a vela. Van Campenhoudt conferma ciò dicendo che in Francia fino al Settecento c'è una differenza marcata tra i due domini linguistici: marineria occidentale (Mare del Nord, Manica, Oceano Atlantico) con l'influenza del gruppo germanico delle lingue, in particolare olandese e le lingue scandinave, e marineria d'Oriente o Levante, dov'è evidente influsso dell'ambiente mediterraneo e i suoi linguaggi, particolarmente occitano d'origine italiana. La scomparsa di galere ha portato alla rapida scomparsa della terminologia utilizzata al loro bordo e nel Mediterraneo (poiché le galere erano navi prevalentemente del Mediterraneo). Questa è la causa per la quale suddetta terminologia non ha lasciato un segno particolare nell'ulteriore evoluzione della terminologia marinara francese.

L'istituzione dell'Accademia di Francia nel 1635, il cui obiettivo principale era la salvaguardia e perfezionamento della lingua francese, quindi la fondazione di Imprimerie Royale - Stamperia Reale nel 1640, testimonia la cura e l'importanza attribuita alla lingua. In questo contesto storico in Francia sono stati stampati numerosi dizionari marinari, a partire dal Seicento, o più precisamente dal 1636 quando è stato pubblicato il primo (Cleirac). Nel 1687 Desroches compone Dictionnaire des termes propres de marine, dizionario più voluminoso rispetto al precedente con 576 pagine e circa 2500 termini in ordine alfabetico. Fino a metà dell'Ottocento ne è stato pubblicato un gran numero. ${ }^{9}$

Al contrario, in Italia, dopo L'Armata navale di Pantero Pantera, stampato a Roma nel 1614, non ci sono state opere significative alla marineria o qualche vocabolario relativo, fino alla traduzione dal francese del Dizionario Istorico,

\footnotetext{
7 II mondo mediterraneo ha determinato il modo di vita imponendo la propria universalità del mondo marittimo e riflettendosi anche nel linguaggio (Božanić et al., 2011).

8 „Par définition plus internationale qu'aucun autre vocabulaire technique, la terminologie maritime s'est nourrie à d'innombrables sources. " (dal francese hanno tradotto autrici)

9 Dictionnaire de marine de Nicolas Aubin (1702)
}

Dictionnaire historique, théorique et pratique de Marine de A. Savérien (1758)

Dictionnaire des termes de marine de Bourdé de Villehuet (1773)

Dictionnaire de la marine française de Charles Romme (1792-1813)

Dictionnaire de Marine de Willaumez (1820-1831)

Dictionnaire pittoresque de marine de Jules Lecomte (1835)

Dictionnaire abrégé de Marine (1834) de Bonnefoux

Dictionnaire de la marine à voile de Bonnefoux et Paris (1848-1859-1885).

Glossaire nautique de Jal (1848) (Marine Nationale, 2011) 
Teorico, e Pratico di Marina del 1769 di Saverien. Questo ampio lavoro permette di comprendere meglio la terminologia relativa alla marineria e si occupa anche della sua storia presentandoci teoria e pratica di quest'arte. Un altro dizionario importante è il Vocabolario di Marina in tre lingue - Italiano, Inglese e Francese del 1813 di Simone Stratico, considerato il migliore del suo tempo. Nell' Ottocento merita di essere menzionato anche Dizionario di marineria militare italiano francese e francese - italiano del 1866 di Parilli, apprezzato principalmente per la parte tecnica, e il Dizionario di marina: italiano francese e francese italiano del 1870 di Fincati. ${ }^{10}$

La lingua letteraria italiana è basata sulla parlata toscana. Questa è la lingua standard che non dispone di espressioni specializzate o di un dizionario del linguaggio marinaro perché la situazione è simile a quella dei Croati nell'Ottocento. Espressioni marinare specialistiche sono state create in idiomi locali, da persone che, parlando ognuna il proprio dialetto, commerciavano, navigavano, costruivano le navi. Loro sono ideatori e portatori di termini nautici che perciò riflettono caratteristiche dialettali. Da qui deriva anche la nota di Simone Stratico - ogni termine deve essere descritto precisamente per poter riconoscere come è stato ideato (Stratico, 1813).

\section{I termini colloquiali e i loro equivalenti nelle fonti francesi e italiani}

Abbiamo scelto la terminologia colloquiale in uso ancora oggi con maggiore o minore frequenza sulla costa adriatica croata (in base alla comunicazione con ufficiali marittimi, studenti di nautica e pescatori). Il corpus contiene 35 lessemi relativi a ordini di bordo, nomi delle parti della nave, corda e velatura. II primo compito è stato quello di stabilire l'esistenza di termini selezionati nel dizionario marittimo di Radovan Vidović, opera la cui materia comprende il periodo dal Medioevo alla Rivoluzione Industriale, cioè periodo di barche a remi e a vela. II materiale per il dizionario, includendo localismi registrati, è stato raccolto da circa 60 vilaggi, dall'Istria alla Baia di Kotor e, come dice l'autore, proviene da una varietà di dialetti. ${ }^{11}$ || secondo scopo è stato esaminare se ci sono equivalenti di questi termini in alcuni vocabolari francesi del Settecento e Ottocento e in quelli italiani dell' Ottocento e Novecento, ignorando l'eventuale presenza di polisemia, e questo come segue:

- Dictionnaire historique, théorique et pratique de Marine de A. Savérien (1758)

- Dictionnaire abrégé de Marine: contenant la traduction des termes les plus usuels en anglais et en espagnole de Bonnefoux (1834)

- Glossaire nautique de Jal (1848)

- $\quad$ Dizionario del dialetto veneziano, III edizione di Boerio (1867)

Tabella 1. Lessemi colloquiali e i loro equivalenti nei dizionari

\begin{tabular}{|c|c|c|c|c|c|c|}
\hline $\begin{array}{c}\text { Termine } \\
\text { colloquiale }\end{array}$ & $\begin{array}{c}\text { Dizionario } \\
\text { marittimo di } \\
\text { Vidović } \\
1984\end{array}$ & $\begin{array}{c}\text { Dictionnaire } \\
\text { de Saverien } \\
1758\end{array}$ & $\begin{array}{c}\text { Dictionnaire de } \\
\text { Bonnefoux } \\
1834\end{array}$ & $\begin{array}{c}\text { Glossaire de } \\
\text { Jal 1848 } \\
\text { (termine } \\
\text { francese) }\end{array}$ & $\begin{array}{c}\text { Glossaire de } \\
\text { Jal 1848 } \\
\text { (termine } \\
\text { italiano) }\end{array}$ & $\begin{array}{c}\text { Dizionario } \\
\text { del dialetto } \\
\text { veneziano di } \\
\text { Boerio 1867 }\end{array}$ \\
\hline ankora & ankora & ancre & ancre & ancre & ancora & ancora \\
\hline asta & ašta & $I$ & $\begin{array}{c}\text { aste, } \\
\text { mediterran. }\end{array}$ & $\begin{array}{c}\text { aste de } \\
\text { bandière }\end{array}$ & asta & asta, aste \\
\hline barbeta & barbita & $I$ & $\begin{array}{c}\text { barbet, } \\
\text { barbette } \\
\text { u drugačijem } \\
\text { značenju) }\end{array}$ & $\begin{array}{c}\text { barbet, } \\
\text { barbette }\end{array}$ & barbetta & barbeta, \\
barbette \\
\hline bigota & bigot, bigota & bigot & $\begin{array}{c}\text { bigots, } \\
\text { mediterran. }\end{array}$ & bigote & bigota, venit. & bigota \\
\hline bokaporta & bokaporta & $l$ & $l$ & & $\begin{array}{c}\text { boccaporta, } \\
\text { ital. } \\
\text { boccaporto, } \\
\text { venit. }\end{array}$ & bocaporta \\
\hline
\end{tabular}

\footnotetext{
${ }^{10}$ Nonostante il gran numero di dizionari ne mancava uno che si adattasse agli specialisti e al pubblico in generale. Dizionario di marina medievale e moderno stampato nel 1937 è il più voluminoso lavoro lessicografico fino ad allora. Oltre alla ricchezza di terminologia marinara corrente con abbondanza di termini dialettali, si presenta anche l'etimologia del termine (Prefazione di Giulio Bertoni, XI-XII). 11 „Nella nomenclatura di questo dizionario italianismi sono rappresentati con più del 80 per cento, e, in questo senso, il dizionario rappresenta un saggio di italianismi nella nostra lingua marittima" (Stepanić, 2005: 252, citato da Boris Pritchard, 1993: 484).
} 


\begin{tabular}{|c|c|c|c|c|c|c|}
\hline $\begin{array}{l}\text { bulina, } \\
\text { burina }\end{array}$ & burina & bouline & bouline & $\begin{array}{l}\text { boline, } \\
\text { bouline }\end{array}$ & $\begin{array}{c}\text { bolina, ital. } \\
\text { borina, burina, } \\
\text { venit. }\end{array}$ & $\begin{array}{l}\text { borina, } \\
\text { bolina }\end{array}$ \\
\hline bumpreš & bumpreš & beaupré & beaupré & beaupré & $\begin{array}{l}\text { bompresso, } \\
\text { buon presso }\end{array}$ & $\begin{array}{l}\text { bompresso, } \\
\text { buompresso }\end{array}$ \\
\hline cima & cima & 1 & 1 & 1 & cima & cima \\
\hline gavun & gavun & $\begin{array}{l}\text { gavon, } \\
\text { terme de } \\
\text { galere }\end{array}$ & l & gavon & gavone & I \\
\hline kaić & kaić & $\begin{array}{c}\text { caique, } \\
\text { en usage au } \\
\text { Levant \& } \\
\text { particulièreme } \\
\text { nt chez les } \\
\text { Turcs. }\end{array}$ & caique, Levant & $\begin{array}{l}\text { caïc, caïq, } \\
\text { caïque }\end{array}$ & caico, caicchio & $\begin{array}{c}\text { caichio, } \\
\text { caechio, } \\
\text { caicco }\end{array}$ \\
\hline kolumba & kolumba & 1 & 1 & I & kolumba & colomba \\
\hline korpomorto & korpomorto & corps mort & corps mort & corps mort & I & $\begin{array}{c}\text { a corpo } \\
\text { morto }\end{array}$ \\
\hline kuverta & kuverta & $\begin{array}{l}\text { couverte, } \\
\text { terme du } \\
\text { Levant }\end{array}$ & couverte & $\begin{array}{l}\text { couverte, } \\
\text { coverte }\end{array}$ & coperta & $\begin{array}{l}\text { coverta, } \\
\text { coperta }\end{array}$ \\
\hline lantina & lantina & $\begin{array}{l}\text { l'antenne, } \\
\text { mots de } \\
\text { Levantins }\end{array}$ & $\begin{array}{l}\text { l'antenne, } \\
\text { mediterran. }\end{array}$ & l'antenne & l'antena & l'antenna \\
\hline madijer & $\begin{array}{c}\text { madijer }= \\
\text { madir, madiri }\end{array}$ & madiers & madrier & madier & $\begin{array}{c}\text { matera, } \\
\text { mader, venit. }\end{array}$ & mader \\
\hline manovra & manovra & manoeuvre & manoeuvres & manoeuvre & manovra & manuvra \\
\hline matafjuni & matafuni & matafions & 1 & 1 & matafione & 1 \\
\hline merlin & merlin, mrlin & merlin & merlin & merlin, & merlino & merlin \\
\hline noštromo & noštromo & I & $\begin{array}{c}\text { nostr'homme } \\
\text { mediterran. }\end{array}$ & notre homme & nostro homo & nostromo \\
\hline pajol & pajol & paillot & $\begin{array}{c}\text { payol, } \\
\text { mediterran. }\end{array}$ & paillot, payol & I & $\begin{array}{l}\text { pagiol, } \\
\text { pagiola }\end{array}$ \\
\hline pašteka & pašteka & I & $\begin{array}{c}\text { pastèque, } \\
\text { passetèque } \\
\text { mediterran. }\end{array}$ & pastèque, & $\begin{array}{l}\text { pastecca, } \\
\text { pasteca, } \\
\text { pasteco }\end{array}$ & pasteca \\
\hline prova & prova & proue & proue & proue & prova, venit. & prova \\
\hline sartije & sartija, sartije & $\begin{array}{c}\text { sartie, } \\
\text { méditerran. }\end{array}$ & sartis & sartie, sartis & sartia & $\begin{array}{c}\text { sarchie, } \\
\text { sartie, sarte }\end{array}$ \\
\hline škota & škota & 1 & 1 & scote, & scotta & scota, scotta \\
\hline štiva & štiva & 1 & 1 & I & stiva & stiva \\
\hline timun & timun & timon & timon & timon & timon, venit. & timon \\
\hline trcaroli & $\begin{array}{l}\text { trcarol, } \\
\text { trcaroli }\end{array}$ & I & I & tiercerot & $\begin{array}{c}\text { terzaruolo, } \\
\text { terzarolo, } \\
\text { terzeruolo }\end{array}$ & 1 \\
\hline $\begin{array}{l}\text { trinket, } \\
\text { trinketa }\end{array}$ & $\begin{array}{l}\text { trinket, } \\
\text { trinketa }\end{array}$ & $\begin{array}{l}\text { trinquet, } \\
\text { trinquette }\end{array}$ & $\begin{array}{l}\text { trinquet, } \\
\text { trinquette }\end{array}$ & $\begin{array}{l}\text { trinquet, } \\
\text { trinquette }\end{array}$ & trinchetto & trincheto \\
\hline $\begin{array}{l}\text { Comandi } \\
\text { dai verbi }\end{array}$ & $\begin{array}{l}\text { Dizionario } \\
\text { marittimo di } \\
\text { Vidović } \\
1984\end{array}$ & $\begin{array}{c}\text { Dictionnaire } \\
\text { de Saverien } \\
1758\end{array}$ & $\begin{array}{c}\text { Dictionnaire de } \\
\text { Bonnefoux } \\
1834\end{array}$ & $\begin{array}{c}\text { Glossaire de } \\
\text { Jal } 1848 \\
\text { (termine } \\
\text { francese) }\end{array}$ & $\begin{array}{l}\text { Glossaire de } \\
\text { Jal } 1848 \\
\text { (termine } \\
\text { italiano) }\end{array}$ & $\begin{array}{c}\text { Dizionario } \\
\text { del dialetto } \\
\text { veneziano di } \\
\text { Boerio } 1867\end{array}$ \\
\hline akoštat & $\begin{array}{c}\text { akoštat, } \\
\text { koštat }\end{array}$ & $\begin{array}{l}\text { accoster, } \\
\text { mediterran. }\end{array}$ & 1 & $\begin{array}{l}\text { accoster, } \\
\text { acoster }\end{array}$ & $\begin{array}{l}\text { accostare, ital. } \\
\text { accostar, venit. }\end{array}$ & $\begin{array}{l}\text { acostar, } \\
\text { Acosta! }\end{array}$ \\
\hline $\begin{array}{l}\text { mainat, } \\
\text { majnat }\end{array}$ & $\begin{array}{l}\text { mainat, } \\
\text { majnat }\end{array}$ & 1 & 1 & I & $\begin{array}{c}\text { mainar, } \\
\text { mainare, venit. }\end{array}$ & $\begin{array}{l}\text { mainar, } \\
\text { Maina! }\end{array}$ \\
\hline
\end{tabular}




\begin{tabular}{|c|c|c|c|c|c|c|}
\hline molat & molat & molir & mollir & mollir & molare & molar \\
\hline orcat & orcat & $\begin{array}{c}\text { orser, } \\
\text { terme du } \\
\text { Levant }\end{array}$ & $\begin{array}{c}\text { orse, m., } \\
\text { mediterran. }\end{array}$ & orser, & $\begin{array}{c}\text { orzare, orsare, } \\
\text { orzeggiare }\end{array}$ & Orza! \\
\hline $\begin{array}{c}\text { pođat, } \\
\text { pojat }\end{array}$ & pojat & $\begin{array}{c}\text { Poge!, } \\
\text { terme } d u \\
\text { Levant }\end{array}$ & $/$ & À poge! & $\begin{array}{c}\text { poggiare, ital. } \\
\text { poggiar, venit. }\end{array}$ & Pogia! \\
\hline šijat & šijat & scier à caler & scier & scier & $\begin{array}{c}\text { sciare, ital. } \\
\text { siare, venit. }\end{array}$ & $\begin{array}{c}\text { siar, sciar, } \\
\text { Sia!, Scia! }\end{array}$ \\
\hline virat & virat & virer & virer & virer & virare & virar, Vira! \\
\hline
\end{tabular}

Dopo aver confrontato gli equivalenti abbiamo concluso quanto segue:

- Tutti i lessemi citati sono registrati nel dizionario marittimo di R. Vidović

- nel Dictionnaire historique, théorique et pratique de Marine de A. Savérien sono stati trovati 24 equivalenti; nel Dictionnaire abrégé de marine de Bonnefoux anche; nel Glossaire nautique de Jal 29 equivalenti nella lingua francese e 33 nella versione italiana dello stesso dizionario; nel Dizionario del dialetto veneziano sono stati notati 32 equivalenti dei lessemi marinari colloquiali

Già dal primo sguardo alla tabella 1 diventa chiaro che gli equivalenti citati sono adattati in lingua croata a livello fonologico e sono riconoscibili dagli appartenenti di diverse nazioni. Che si tratta di mediterraneismi è suggerito anche dagli autori dei singoli dizionari come Bonnefoux che per sei lessemi menziona la loro utilizzazione nel Mediterraneo (e offre il suo sinonimo invece della spiegazione, per esempio pastèque = syn. galoche), e per un termine afferma la sua provenienza dal Levante. Saverien tra i lessemi osservati annota cinque provenienti dal Levante e due che sono di origine mediterranea. Intanto, nei dizionari francesi non sono trovati equivalenti per cinque termini: bokaporta, cima, kolumba, štiva e per il comando Maina, mentre nelle fonti italiane sono presenti tutti i lessemi scelti. Ė importante notare, inoltre, che Jal per alcuni nomi registrati segna la versione italiana e in particolare veneta. Oltre al modello di Boerio questo conferma che la maggior parte delle espressioni colloquiali marinare sono versioni coloniali del dialetto veneziano. „Il contrasto linguistico tra Venezia e Dalmazia, secondo i filologi americani, è meno importante del fatto che esista lo spazio culturale Adriatico (Adriatic cultural area), che in gran parte ha creato una propria espressione marittima. Questo è il motivo per cui le differenze regionali tra Venezia, l'Istria e la Dalmazia furono irrilevanti ai marinai: alcune parole erano semplicemente parole adriatiche (Adriatic words)" (Stepanić, 2005: 251 secondo H. Kahane, R., Kahane e Koshansky, 1953/54: 160-161).12

Per tal motivo la maggior parte di questi lessemi troverebbe un posto nell'Atlante linguistico del Mediterraneo (Deanović, 1962), ${ }^{13}$ progetto a lungo pianificato ma mai realizzato. Era un'idea di molte popolazioni che, vivendo in tre continenti e condividendo la stessa visione e lo stesso mare, hanno fortemente influenzato la civiltà e la storia europea e mondiale. Perciò la terminologia croata colloquiale appartiene all'espressione mediterranea marittima comune che si è mantenuta, con certe trasformazioni, sulle nostre coste fino ad oggi. ${ }^{14}$

\section{6. È necessaria la conservazione della terminologia colloquiale?}

Con la standardizzazione dei neologismi e l'introduzione di parole coniate in lingua croata, le espressioni colloquiali adriatiche vengono soppresse e si incontrano raramente in dizionari croati o di solito sono indicate come regionalismi.

\footnotetext{
12 "Quelle parole spesso sono state caratterizzate come veneziane d'oriente (East Venetian), corrispondenti al dialetto italiano usato dai marinai austriaci. È chiaro il riferimento ai marinai croati della costa orientale dell'Adriatico, che allora faceva parte della monarchia austro-ungarica" (Stepanić, 2005: 252).

${ }_{13}$ L'autore presenta un esempio di questionario pubblicato nel 1960, che comprende una serie di domande relative a diversi settori di attività umana - costruzione navale, pesca, navigazione, tipi di navi, ecc.

${ }^{14}$ Dagli anni novanta del Novecento, questa espressione spesso viene chiamata lingua franca. Ma, siccome si tratta di una lingua artificiale, morta, e non parlata mediterranea viva, cresciuta al contatto diretto con il dialetto veneto, dovrebbe essere notato che l'espressione dalmata dialettale è la versione della lingua coloniale veneziana che non va confusa con linguaggio artificiale utilizzato per il commercio tra gli Europei, Arabi e Turchi sulle coste africane del Mediterraneo, con il lessico costituito principalmente da elementi italiani e spagnoli (Stepanić, 2011: 164-165). L'attualità della lingua franca negli ultimi cento anni è rafforzata dall' interesse dei linguisti a causa delle diverse interpretazioni del termine franco in questa espressione (Viarello, 1955: 67).
} 
L'eccezione è il già citato dizionario maritimo di Radovan Vidović in cui è stato registrato un gran numero di espressioni colloquiali e dialettali.

In tempi recenti, i linguisti croati sottolineano l'importanza di registrazione della terminologia colloquiale viva, che testimonia la ricchezza dell'espressione croata marinara. ${ }^{15}$ Così, la Fondazione nazionale per la scienza, l'istruzione superiore e lo sviluppo tecnologico della Repubblica di Croazia ha riconosciuto l'importanza di stabilire la terminologia specialistica e di specificare la terminologia usuale. Essa ha iniziato un progetto per creare una terminologia professionale croata - STRUNA. ${ }^{16}$ In STRUNA sono stati notati i seguenti termini presentati nella tabella 1, ma etichettati con lo stato normativo di gergo: ankora, bokaporta, korpomorto, lantina, pašteka, škota, štiva e orcat. È registrato anche bumpreš marcato come „termine archaico". Dato che la maggior parte dei lessemi colloquiali fanno parte del vocabolario attivo, questo numero è relativamente piccolo. Ciò è confermato dai risultati di un sondaggio condotto nella zona di Dubrovnik nel 2009 (Stepanić, Violić-Koprivec e Maslek: 2009). Gli ufficiali marittimi intervistati, per lo più capitani di lungo corso di Dubrovnik, per kolumba, manovra e škota non conoscevano il termine standard. Allo stesso modo, agli studenti del primo anno del Dipartimento marittimo all'Università di Dubrovnik non era noto il termine standardizzato della parola colloquiale štiva. Guardando la frequenza di uso secondo questa indagine, è importante notare che gli ufficiali marittimi usano regolarmente korpomorto e pajol, e molto spesso vengono utilizzati prova, šijat, škota, trcarol, bokaporta, kolumba, manovra, orcat, sartija e virat. Dall'altra parte, gli studenti usano spesso prova, cima e molat. II fatto è che un certo numero di neologismi non ha preso piede, mentre i loro sinonimi colloquiali non si trovano nei dizionari della lingua croata. Questo è confermato da Božanić, Lozić Knezović, Runjić-Stoilova e Tomelić Ćurlin (2011: 49): „Certe parole, come kolumba, prova, šešula, škota, timūn, vala, sono marcate come regionalismi, anche se i loro neologismi standardizzati non sono mai stati accettati dal cerchio marittimo culturale croato e non sono arrivati all'esperienza viva." Inoltre, gli autori confermano una mancanza di termini standardizzati adeguati in lingua croata contemporanea, relativi alla terminologia marinara.

II fallimento che in pratica ha subito il tentativo di nazionalizzare la nostra terminologia marittima purificandola radicalmente, in particolare la nomenclatura tecnica della nave e la velatura delle navi a remi, è stato causato principalmente del fatto che i fautori e gli esecutori di questo atteggiamento non avevano capito che non si trattava solo di una questione di nomenclatura tecnica. Si trattava di una cultura formata nel contatto con il mare e con culture vicine abbracciate dallo stesso mare. E l'Adriatico e la cultura marittima croata, che per secoli aveva creato il suo ricchissimo vocabolario marittimo ereditando la cultura e la terminologia principalmente dalmatica, poi veneta e mediterranea [...] Considerare quello strato lessicale straniero, per lo più di origine italiana, significa e rinunciare alla propria cultura che si era sviluppata per secoli dal contatto con culture vicine, cioè dal contatto del popolo croato con l'universo culturale e linguistico del Mediterraneo (Božanić et al., 2011: 49).

\section{Conclusione}

I lessemi colloquiali selezionati hanno confermato l'esistenza di espressioni costiere marittime in Croazia, costituiti dalla parlata viva che ha avuto origine in contatto con culture vicine e che appartiene al circolo culturale e linguistico del Mediterraneo. Se respingiamo tale idioma nella lessicografia sostituendolo con quello di nuova formazione, impoveriamo automaticamente l'espressività e la particolarità di espressione, che per più secoli faceva parte della cultura marittima croata. I mediterraneismi universali nella nostra comunicazione colloquiale dovrebbero trovare posto nei dizionari della lingua croata, accanto alla terminologia standardizzata, come nostri, e non come terminologia marinara altrui.

\section{Fonti}

Dictionnaire historique, théorique et pratique de Marine de A. Savérien (1758)

Dictionnaire abrégé de Marine: contenant la traduction des termes les plus usuels en anglais et en espagnole de Bonnefoux (1834)

Glossaire nautique de Jal (1848)

Dizionario del dialetto veneziano, III edizione, Venezia (1867) di G. Boerio.

\footnotetext{
15 Vedere Stolac, 2010.

${ }^{16}$ STRUNA è la base terminologica della terminologia croata specializata in cui si raccoglie, produce, elabora e sistematicamente interpreta la terminologia di diverse professioni per riunire e armonizzare la terminologia croata specialistica. In STRUNA finora sono incluse diciannove diverse professioni e al pubblico è accessibile la terminologia di tredici professioni, tra loro anche quella marinara (STRUNA, 2013).
} 
Vocabolario di marina in tre lingue Ital.-Franc.-Ingl., sv.1. Milano (1813) di S. Stratico

Dizionario di marina medievale e moderno, Roma (1937)

Pomorstvo, godište XXVIII broj 5-6 (1973).

\section{Bibliografia}

Académie française. Du françois au français-Naissance et évolution du français, <http:www.academie-francaise.fr/la-languefrancaise/le-francais-aujourdhui> (pristup: 15. veljače 2013.)

Bertoša, M. (2002). Kada svijet bijaše mediteranski. Hrvatska i Sredozemlje od XVI. do XVIII. stoljeća. Povijesni i kulturnoantropološki nacrt. U M. Bertoša, Izazovi povijesnog zanata. Lokalna povijest i sveopći modeli. (str. 19-58). Zagreb: Antibarbarus.

Bloomfield, L. (1970). Language. London: George Allen \& Unwin.

Božanić, J., Lozić Knezović K., Runjić-Stoilova A., \& Tomelić Ćurlin M. (2011). Leksička norma u hrvatskoj leksikografiji 20. stoljeća. Filologija, 57, 35-51.

Carić, J. (1890). Nautika (geodetični dio), udžbenik.

Deanović, M. (1962). Linguistica e storia: L'Atlante linguistico mediterraneo. estratto da: Mediterraneo e oceano indiano, Atti del VI Collooquio Internazionale di Storia marittima, Venezia: 20-29 Settembre, 310- 316.

Hrvatsko strukovno nazivlje - projekt koordinacije (STRUNA), Nacionalna zaklada za znanost - Institut za hrvatski jezik i jezikoslovlje. $<$ http://struna.ihjj.hr/> (pristup: 15. ožujka 2013.)

Jernej, J. (1956). Sugli italianismi penetrati nel serbo-croato negli ultimi cento anni. Studia Romanica, I (1), 54-82.

Kahane, H., Kahane R., \& Koshansky, O. (1953/54). Venetian Nautical Terms in Dalmatia. Romance Philology, VII (2-3), 156-170.

Lovrić-Jović, I. (2006). Fonološka adaptacija talijanizama u dubrovačkim oporukama iz 17. i 18. stoljeća. Rasprave Instituta za hrvatski jezik i jezikoslovlje, 32, 173-192.

Marine nationale. http://www.defense.gouv.fr/marine/decouverte/patrimoine/traditions/lexique/lexique (pristup: 12. veljače 2013.)

Muljačić, Ž. (2007). U potrazi za starijim hrvatskim pseudoarabizmima. Suvremena lingvistika, 64, 159-178.

Pritchard, B. (1993). Povijesni razvitak hrvatske jezične leksikografije u pomorstvu. Pomorski zbornik, 31, 471-489.

Pritchard, B. (1997). O ranom razdoblju hrvatske jezične leksikografije u pomorstvu. Riječ, 1, 57-65.

Sočanac, L. (2002). Talijanizmi u hrvatskome jeziku. Suvremena lingvistika, 53-54, 127-142. Sočanac, L. (2004). Hrvatsko-talijanski jezični dodiri: s rječnikom talijanizama u standardnome hrvatskom jeziku i dubrovačkoj dramskoj književnosti, Zagreb: Nakladni zavod Globus.

Stepanić, Ž. (2005). Hrvatsko pomorsko nazivlje od početaka do polovine 19. stoljeća. Naše more, 52 (5-6), $248-257$.

Stepanić, Ž. (2006). Hrvatsko pomorsko nazivlje od polovine 19. stoljeća do sloma Austro-Ugarske Monarhije. Naše more 53(1-2), 6376.

Stepanić, Ž., Violić-Koprivec, A., \& Maslek, J. (2009). Kolokvijalno pomorsko nazivlje na dubrovačkom području. Naše More, 5-6, 225236.

Stepanić, Ž. (2011). Postoji li dalmatinska lingua franca? Naše more 58 (3-4), 162-171.

Stolac, D. (1998). Hrvatsko pomorsko nazivlje (Božo Babić, njegovi prethodnici i nastavljači). Rijeka: Izdavački centar Rijeka.

Stolac, D. (2010).Tehničko nazivlje između norme i uzusa, Zbornik radova petoga hrvatskog slavističkog kongresa, 511-522.

Stulli, B. (1953). O hrvatskom jeziku u riječkoj Nautici i radu J. A. Mikoča, Rijeka: Riječka revija.

Urbany, M. (1996). Restriktivne oznake u leksikografiji hrvatskoga pomorskog jezika. Zbornik radova Pomorskog fakulteta u Rijeci, 10, 205- 208.

Van Campenhoudt, M. (2002). Conférence Petite introduction au domaine de la terminologie maritime et à I'histoire des dictionnaires de marine et présentation de Dhydro, Bruxelles, Institut libre Marie Haps. <http://www.termisti.org/mdroit1.htm\#articles> (pristup: 20. veljače 2013.)

Viarello, N. (1955). Lingua franca di Barberia e lingua franca di Dalmazia. Lingua nostra, Firenze, 67-69.

Vidović, R. (1982). Povijest rada na sakupljanju i obradi naše pomorske terminologije (od početka do drugoga svjetskog rata). Mogućnosti, 8-9-10, 770-803

Violić-Koprivec, A., Maslek J., \& Leoni L. (2012). Italianismi nella terminologia marinara nella zona di Dubrovnik/Ragusa dall'antichità ad oggi. Raccolta di atti Mediterraneo - Archeologia e civiltà del vino. Produzione, scambi, barche, traffici dall' Antichità ad oggi. Cattolica: Istituzione Culturale della Regina, 157-162. 